\title{
Technology of obtaining fine textured films for femtosecond instrumentation based on photon echo
}

\author{
R.W. Belyaev, A.U. Bakhadurov, N.S. Vashourin, K.Sh. Gazizov, A.N. Musanov, \\ D.A. Nikitin, I.I. Popov*, A.V. Moroz, S.A. Stepanov, and N.I. Sushentsov
}

Volga State Technological University, 3 Lenin sq., Yoshkar-Ola, 424001 Russia

\begin{abstract}
This paper presents the technological approaches to the creation of thin textured films by the method of magnetron sputtering with parameters ensuring excitation of detected photon echo signals in them and the adjusted metrological characteristics of devices on a photon echo.
\end{abstract}

The purpose of this report is to develop and study the technology of obtaining thin textured films for femtosecond instrumentation based on photon echo. The physical foundations of the developed instruments rely on the excitation and use of the properties of the photon echo on exciton and trion states localized on surface defects of thin films. The task of technology is to reproduce systematically the thickness of thin films and the parameters of surface defects based on magnetron sputtering of these films. The parameters of technological regimes of magnetron sputtering of films which affect the reproduction of surface defects and the thickness of these films are reported in the work. Different variants of obtaining single-layer and multilayered textured thin films obtained under various magnetron sputtering regimes are given. Thin structured films obtained by magnetron sputtering methods have a fibrous structure. The Roentgen-amorphous phase fills the gap between the filaments. The surface defects were formed on the surface of individual fibers on which the excited exciton states were localized. The exciton states are described by the wave function typical for the superposition state.

Nearly located defects had closely located exciton lines with a lower binding energy than that of the surface particles of the fibers that do not have defects. They formed a cluster of neighboring particles with surface defects surrounded by an energy barrier of particles that do not have defects (Fig. 1). Because of the localization on the defects, the excitons are strongly bound to the phonons of the lattice and in the absorption and recombination spectra of excitons there is a phonon wing proportional to the density of the phonon states. This leads to a strong inhomogeneous broadening of the exciton lines.

At the interface between the crystalline and amorphous phase, changing various parameters of the technological process, such as the substrate temperature, the sputtering time, the composition of the gas mixture (Fig. 2), the number and composition of layers in

\footnotetext{
* Corresponding author: Popov@volgatech.net
} 
the multilayer film (Fig. 3), and others, there comes the possibility of the controlled introduction of the surface defects. In this paper, methods and means for controlling the production technology of films are proposed, which make it possible to obtain films with a given configuration of surface defects. The films thus obtained are used to demonstrate the physical basis of the principle of constructing a device based on the photon echo.

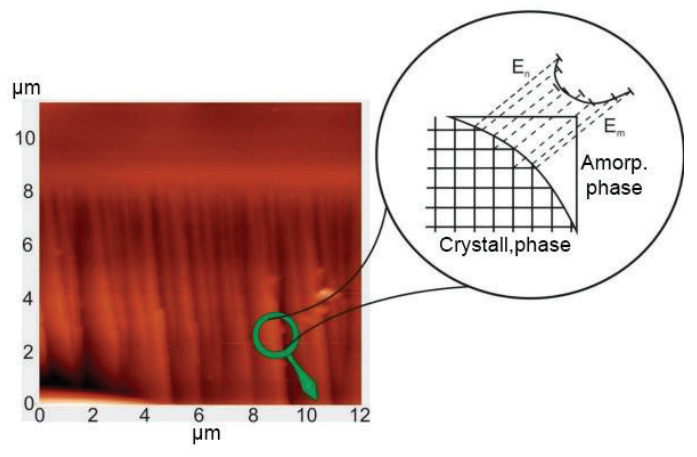

Fig. 1. The image of the cleaved film

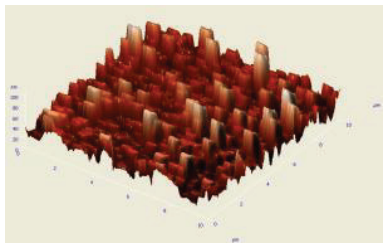

a

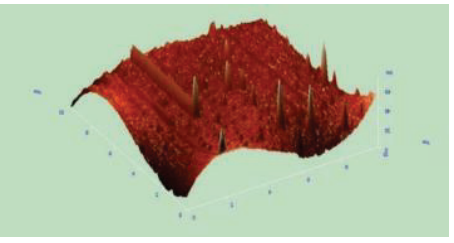

b

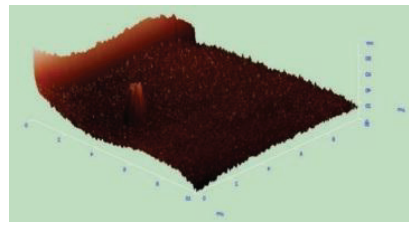

c

Fig. 2. The shapes of the surface and the protruding above it crystalline fibers of the films, when various gases except the sputtered target material were introduced into the chamber, at the moment when a film of $\mathrm{ZnO}$ (a), Si (B), Si (P) was formed.
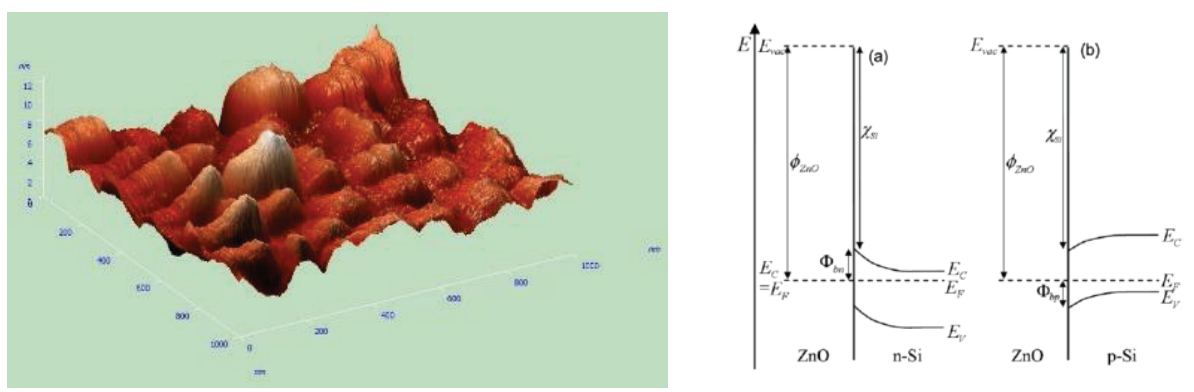

Fig. 3. The shape of the surface and the protruding above it crystal fibers (a) and the band structure of the $\mathrm{ZnO}$ heterojunction with Si n-type and Si p-type for a three-layer film [1].

\section{References}

[1] V. Quemener, M. Alnes, L. Vines, et al., Journal of Physics D 45(31), 315101 (2012) 